\title{
Blooms of a key grazer in the Southern Ocean - An individual-based model of Salpa thompsoni
}

\author{
Jürgen Groeneveld ${ }^{\mathrm{a}, \mathrm{b}, \mathrm{c}, *}$, Uta Berger ${ }^{\mathrm{b}}$, Natasha Henschke ${ }^{\mathrm{d}}$, Evgeny A. Pakhomove, ${ }^{\mathrm{d}, \mathrm{f}}$, \\ Christian S. Reiss ${ }^{g}$, Bettina Meyer ${ }^{\mathrm{h}, \mathrm{i}, \mathrm{j}, *, 1}$ \\ a Department of Economics, Leipzig University and German Centre for Integrative Biodiversity Research (iDiv) Halle-Jena-Leipzig, Deutscher Platz 5e, 04103 Leipzig, \\ Germany \\ ${ }^{\mathrm{b}}$ Institute of Forest Growth and Forest Computer Sciences, Technische Universität Dresden, Piennerst. 8, 01735 Tharandt, Germany \\ ${ }^{\mathrm{c}}$ Helmholtz Centre for Environmental Research - UFZ, Department of Ecological Modelling, Permoserstraße. 15, 04318 Leipzig, Germany \\ ${ }^{\mathrm{d}}$ Department of Earth, Ocean and Atmospheric Sciences, University of British Columbia, Vancouver, BC, V6T 1Z4, Canada \\ ${ }^{\mathrm{e}}$ Institute for the Oceans and Fisheries, University of British Columbia, Vancouver, BC, V6T 1Z4, Canada \\ ${ }^{\mathrm{f}}$ Hakai Institute, P.O. Box 309, Heriot Bay, BC, VOP 1HO, Canada \\ ${ }^{\mathrm{g}}$ Antarctic Ecosystem Research Division NOAA Fisheries, Southwest Fisheries Science Center, La Jolla, CA 92037, USA \\ ${ }^{\mathrm{h}}$ Alfred Wegener Institute Helmholtz Centre for Polar and Marine Research, Section Polar Biological Oceanography, Am Handelshafen 12, 27570 Bremerhaven, Germany \\ ${ }^{\mathrm{i}}$ Institute for Chemistry and Biology of the Marine Environment, University of Oldenburg, Carl von Ossietzky-Straße 9-11, 26111 Oldenburg, Germany \\ ${ }^{\mathrm{j}}$ Helmholtz Institute for Functional Marine Biodiversity at the University of Oldenburg, Ammerländer Heerstraße 231, 26129 Oldenburg, Germany
}

\section{A B S T R A C T}

The Southern Ocean near the Western Antarctic Peninsula (WAP) is strongly affected by climate change resulting in warmer air temperature, accompanied with reduced sea ice coverage, increased sea water temperature and potential changes in the abundances of two key grazer species Salpa thompsoni (salp) and Euphausia superba (Antarctic krill). While salp abundance is hypothesized to increase, krill abundance is hypothesized to decline with dramatic consequences for the entire food web of the Southern Ocean. A better understanding of the biotic interaction between krill and salps and their population dynamics is thus crucial. However, the life cycle of salps is complicated and barely understood. Therefore, we have developed an individual-based model describing the whole life cycle to better understand the population dynamics of salps and the conditions for blooms. The model has been used to explore if and under what conditions the empirical pattern of large variability in observed salp abundances at the WAP, generated by the long-term data of the US Antarctic Marine Living Resources Program (AMLR) can emerge from a small seeding population. The model reproduced this empirical pattern if daily growth rates of oozoids were higher than previously reported for the WAP (mean growth rate for oozoids $\sim 1 \mathrm{~mm} \mathrm{~d}^{-1}$ ) and if growth rates of blastozooids were lower (mean growth rate $\sim 0.2 \mathrm{~mm} \mathrm{~d}^{-1}$ ). The model suggests that a prerequisite for local salp blooms requires a small founding population in early spring. With climate change it has been suggested that more frequent and earlier transport of salps into the WAP or winter survival will occur. Hence, the risk of salp blooms in the WAP is likely to substantially increase. These findings highlight the importance for an improved quantitative understanding of how primary production and the southward advection of salps will be impacted by climate change.

\section{Introduction}

The pelagic tunicate, Salpa thompsoni (salp hereafter), and the Antarctic krill, Euphausia superba (krill hereafter), are key grazers in the Southern Ocean and important elements of the marine Antarctic food web (Atkinson et al., 2004). Historically salps and krill occupied different areas of the Southern Ocean. While salps were more abundant in the food poorer and warmer waters north of the Antarctic Polar Front, krill were more abundant in colder and more productive areas south of the Antarctic Polar Front (Foxton, 1966, Pakhomov and Hunt, 2017).
With rising sea water temperatures and changes in the sea ice dynamics in the Southern Ocean, however, salps may expand their range farther south and interact more often with krill (Atkinson et al., 2004). The fact that salps have a sexual and an asexual reproduction cycle that allows them to reproduce explosively results occasionally in very high salp densities also called blooms (e.g. mean densities up to 3.5 individuals $\mathrm{m}^{-3}$ and higher values in recent years $>18$ individuals $\mathrm{m}^{-3}$ are reported; Loeb and Santora, 2012). Salp blooms may severely reduce the food availability for krill by consuming a substantial amount of the primary production (Dubischar and Bathmann, 1997). In addition, salps

\footnotetext{
* Corresponding authors at: Department of Economics, Leipzig University and German Centre for Integrative Biodiversity Research (iDiv) Halle-Jena-Leipzig, Deutscher Platz 5e, 04103 Leipzig, Germany (J. Groeneveld); Alfred Wegener Institute Helmholtz Centre for Polar and Marine Research, Section Polar Biological Oceanography, Am Handelshafen 12, 27570 Bremerhaven, Germany (B. Meyer).

E-mail addresses: juergen.groeneveld@idiv.de (J. Groeneveld), bettina.meyer@awi.de (B. Meyer).

${ }^{1}$ https://orcid.org/0000-0001-6804-9896.
} 

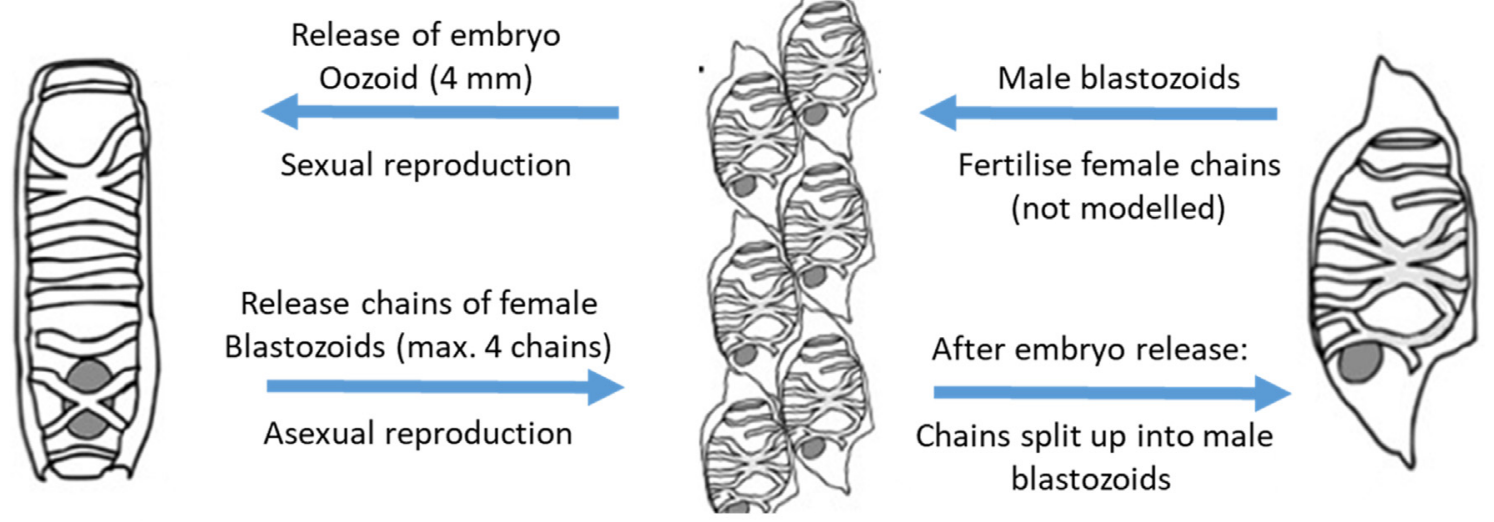

Fig. 1. Modified from Henschke et al. (2018). The life cycle of Salpa thompsoni. Oozoid individuals release chains of female blastozooids. Each female blastozooid can release one oozoid if fertilized. After embryo release the female blastozooid changes sex into a male blastozooid that potentially fertilizes other female blastozooids. We refer to one cycle of sexual and asexual reproduction as one regeneration cycle.

can consume young life stages of krill directly and may therefore increase the mortality rates on krill populations (Huntley et al., 1989). The potential increase of competition between krill and salps may have substantial impact on the krill-dependent Antarctic food web. In contrast to salps, krill are a preferred prey for many marine mammals and seabirds, and are a significant commercial fishery (Nicol and Foster, 2016). Although the contribution of salps as a food resource is not well understood (Pakhomov et al., 2002, Henschke et al., 2016) an increase in salp densities may put the krill-dependent Antarctic food web at risk.

Currently, the population dynamics of salps, particularly in a changing environment, are not well understood and the development of population models for salps and pelagic tunicates have just begun (Henschke et al., 2015a, 2015b, Henschke et al., 2018). Modelling the population dynamics of salps needs to reflect the large variability of salp abundance in space and time. If environmental conditions are suitable, oozoids show a massive asexual reproduction by releasing chains of genetically identical blastozooids (Fig. 1). In contrast, each blastozooid can produce one oozoid embryo during the sexual part of the reproductive cycle. Earlier studies have reported the reproductive cycle (both sexual and asexual parts) lasts between 9 and 12 months, suggesting a single annual production period (Foxton, 1966, Loeb and Santora, 2012). However, recently Pakhomov and Hunt (2017), using an eulerian study, showed that salps were capable of completing two reproductive cycles per year. If this were possible, the per capita growth rate would increase significantly given that one single oozoid may release under optimal conditions more than 800 blastozooids (Daponte et al., 2001). Clearly, resolving the limits of the salp life history is critical to projecting future impacts on the pelagic ecosystem of the Southern Ocean.

To improve our understanding of the population dynamics and salp bloom risk, we have developed an individual-based population model for salps. In contrast to previous pelagic tunicate models, our model aims to reproduce the broad distribution of observed salp abundances at the AMLR sites (US Antarctic Marine Living Resource Program) ranging from 0 to 44.000 individuals $1000 \mathrm{~m}^{-3}$ (Fig. 2a) instead of modelling the average density (Henschke et al., 2018). The US Antarctic Marine Living Resources Program (AMLR) at the WAP offers a unique long-term data set of salp and krill observations and chlorophyll $a$ measurements among other variables (Fig. 2). It covers a large spatial extent from the Brainsfield Strait to the Elephant Island area (see Reiss et al., 2008; Loeb et al., 2010 for more details and a map of the survey grid) between 1996 and 2011. Correlating chlorophyll $a$ and salp counts is challenging because it is often impossible to disentangle positive effects of food on growth and negative effects on food provision due to grazing. The observational data suggest (Fig. 2c) that high salp densities limit chlorophyll $a$ availability although there is large uncertainty. The data also show that salp blooms cannot be found where the summer water temperatures are low (below $-0.5^{\circ} \mathrm{C}$, Fig. $2 \mathrm{~d}$ ). The individualbased model includes the feedback between primary production and salp abundance to limit salp population growth as well as individual variability to better understand the emergence of salp bloom years. We calibrated the model using two population level patterns: (1) the intraannual distribution pattern of salps (2) and the observed large variability of population densities in space and time at the AMLR sites.

From the simulations we observed the population densities, the number of completed life cycles in a season, the oozoid $v s$. blastozooid ratio, the time when a founding population migrated successfully into the simulation area and the individual daily growth rate in length.

\section{Methods}

We calibrated the model to reproduce the variability in salp abundance data from the US Antarctic Marine Living Resource (AMLR) program run by the National Oceanic and Atmospheric Administration (NOAA). During the AMLR program, abundance data of krill and salps as well as environmental data (e.g. chlorophyll $a$ ) were annually collected around the Western Antarctic Peninsula (WAP) between 1996 and 2011 (see Loeb et al., 2010 for details). Surveys were conducted during the austral summer (January-March). We compared our model results with the distribution of all salp abundance observations which ranges over four orders of magnitude (Fig. 2a). We differentiate two model scenarios related to food availability: ((1) Constant food scenario: Each year the same peak chlorophyll $a$ concentration will be reached if no grazing would occur (mean of the observed chlorophyll $a$ concentrations from the AMLR sites). (2) Variable food scenario: The observed chlorophyll $a$ concentrations (Fig. 2b) are used to derive a lognormal distribution that is used in the model to determine the peak chlorophyll $a$ concentration in summer (see sub-model "Chlorophyll dynamics" for more details).

\subsection{Model description}

The model description follows the Overview, Design and Details protocol suggested by Grimm et al. $(2006,2010)$.

\subsection{Purpose}

The purpose of the individual-based model is to describe and project the population dynamics of salps in the Antarctic Peninsula regions of the Southern Ocean. Specifically, we quantify the patterns of intra- 
a)

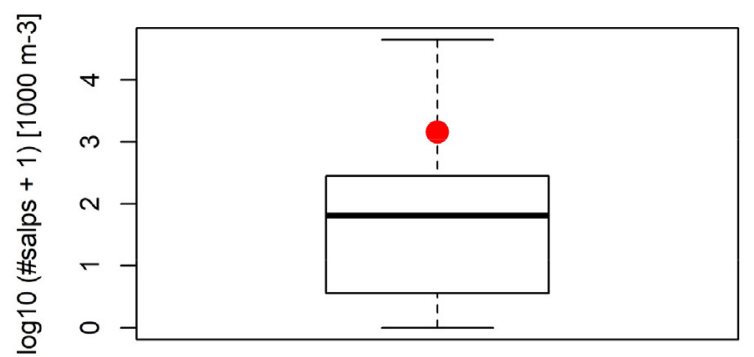

c)

Observed salp abundances at AMLR sites

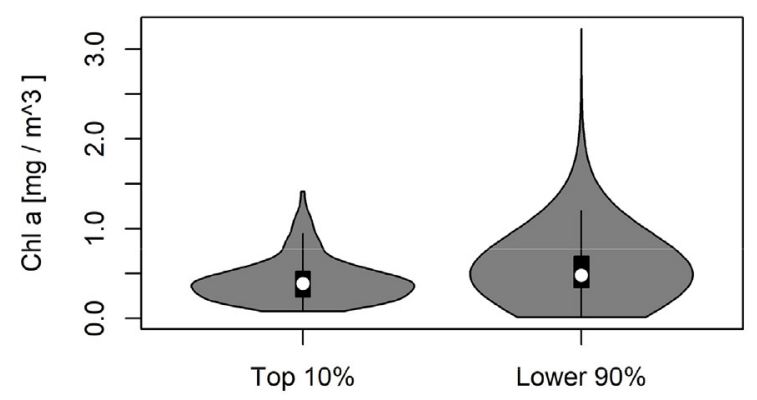

Salp abundance b)

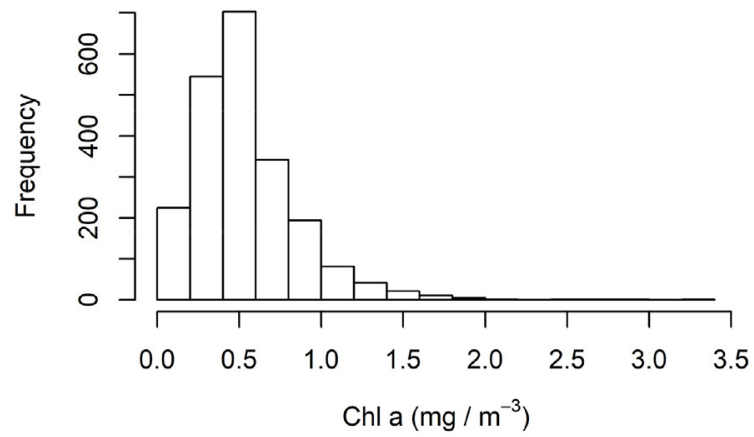

d)

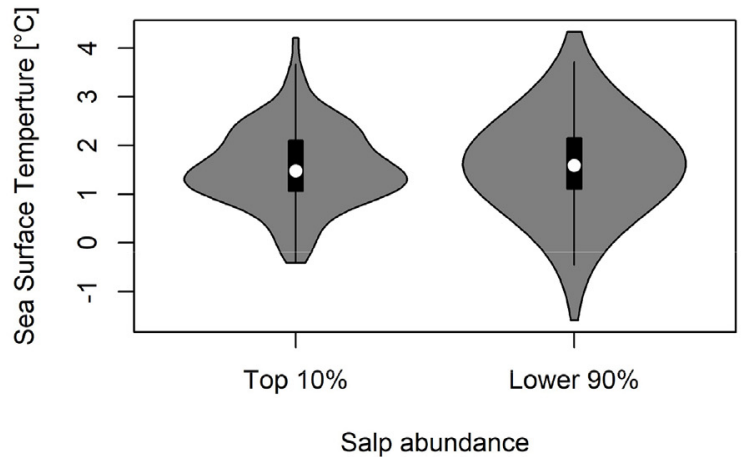

Fig. 2. Overview of the long term data set of the Antarctic Marine Living Research (AMLR) by NOAA that has been used to constrain the model. (A) Distribution of the observed salp densities. The distribution is dominated by observations with no salps observed. If salps have been observed, the number varies over four orders of magnitude with a maximum density of 44,241 salps in $1000 \mathrm{~m}^{3}$ of water. The red point indicates the $95 \%$ quantile of observed densities $\mathrm{q}=1440$ salps $1000 \mathrm{~m}$ (the box presents the range of the second and third quartiles, the line in the box is the median and whiskers show the full range of the data as long as the distance of the extreme points does not exceed 1.5 times the interquartile range from the box). (B) Measured chlorophyll $a$ also varies substantially. C-D Here we present (C) chlorophyll $a$ and (D) sea surface temperature distributions for the highest $10 \%$ salp abundances and the remainder (lower $90 \%$ ). It can be seen that salp abundances are controlled by low temperatures and that high densities of salps have not been observed for very high chlorophyll $a$ content (the area in the violin plot $\mathrm{C}$ and $\mathrm{D}$ indicate the frequency of the observed value).

annual abundance distribution, the inter-annual fluctuations, the number of reproductive cycles per year and the ratio between the oozoid stage and the blastozooid stage. In this study we focus on the local population dynamics on similar spatial resolution as empirical samples have been taken (several $1000 \mathrm{~m}^{3}$ ) in order to understand and predict the enormous variability of salp abundances in space and time in the context of environmental changes as observed in nature. We include three sources of variability which we hypothesize that could lead to the emergence of the observed abundance distributions: (1) demographic stochasticity, (2) variability in food availability and (3) variability in the initial seeding population, i.e. whether and when a small seeding population has been transported into the simulated arena. Furthermore, the model should reflect the measured distribution of daily length growth and therefore a pattern on the individual level. Since the model will be eventually linked to a krill model in the near future, the longterm purpose of the salp model is also to develop a simulation tool for assessing the potential interaction of the two key grazers of the Southern Ocean.

\subsection{Entities, state variables, and scales}

The model has four entities: patches, and three kinds of salp individuals: oozoids, chains of female blastozooids and male blastozooids. Patches have the state variable chla (density of chlorophyll $a$ measured in $\mathrm{mg}$ chlorophyll $a \mathrm{~m}^{-3}$ ) and the state variable pchla (total amount of chlorophyll $a$ in one patch measured in mg chlorophyll $a$ ) as a proxy for the available food for salps. Oozoids, chains and male blastozooids have some state variables in common such as: length $l$, age $a$, number of individuals $n$ (which is the number of blastozooids in one chain and always one for oozoids and male blastozooids), and the accumulated number of days without sufficient food, $t_{\text {starv }}$. In addition, blastozooids are sex specific, whereas female blastozooids form chains. The temporal resolution in the model is one day. The simulation runs over several years to compile time series. In this paper, the overall run time was 300000 days. The spatial resolution is 16 cubic meters and the simulation arena is a layer of $13 \times 13=169$ patches resulting in a simulated volume of $2704 \mathrm{~m}^{-3}$. Thus, we assume that each patch, which is the NetLogo term for the smallest spatial resolution in the model, is representing a volume of 16 cubic meters like a cube with edge length of roughly $2.5 \mathrm{~m}$. The amount of food in one patch pchla is therefore pchla $=16 \mathrm{~m}^{3} \times$ chla mg chlorophyll $a \mathrm{~m}^{-3}$. The simulated world is a torus, i.e. individuals that leave the simulation arena at one edge will reappear from the opposite edge. This is a technical procedure to avoid edge effects and does not aim to represent the real transport processes. Underlying these periodic boundary conditions is the assumption that the simulation arena is part of a larger spatial domain with the same environmental conditions and salp densities. The model has been implemented in NetLogo version 6.0.3 (Wilensky, 1999) and model analysis were performed using the R Software (R Core Team, 2019).

\subsection{Process Overview and scheduling}

An overview of all processes in the model can be found in Fig. 3. Each daily time step begins with all salps exploiting the resources at 


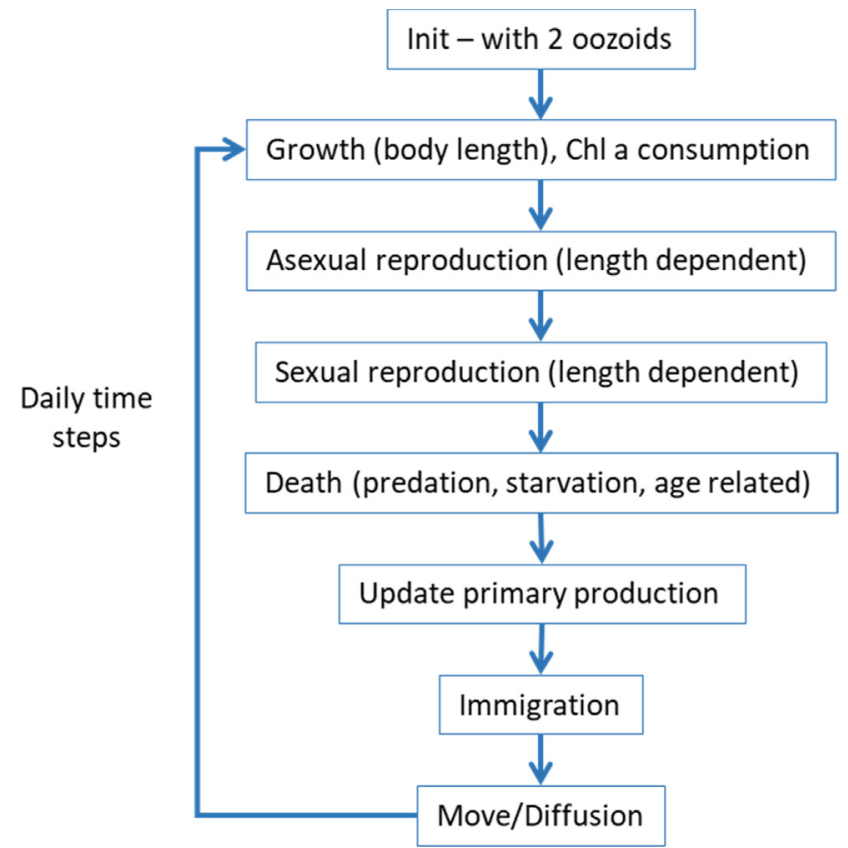

Fig. 3. Flow Chart of the model. Each day the following processes are executed: each salp potentially grows in body length and reduces chlorophyll $a$ availability in its patch, if body length exceeds a certain threshold oozoids will reproduce asexually and blastozooids will reproduce sexually, mortality is determined, the chlorophyll $a$ concentration in each patch is updated (Update primary production), immigration is modelled, and the spatial distribution of salps is updated (Move/Diffusion). See the section "Submodels" for further details.

their location (patch). Next, the daily growth in body length is determined for each salp followed by an asexual or sexual reproduction event if the individual has reached a particular length. Then, the mortality of salps is applied, followed by updating primary production for each patch. If salp abundance is zero in the simulation arena, some immigration of salps from outside is allowed with an immigration probability. To address the uncertainty of the immigration probability we have run the model for different immigration probabilities ranging from 0.001 to 1 . Finally, each salp moves randomly to one of its neighboring patches representing a random walk. All procedures are repeated every time step for each salp. During the course of the year primary production and the vonBertalanffy growth rate in body length changes whereas all other parameters remain constant during the year (e.g. mortality).

\subsection{Design principles}

\subsubsection{Basic principles}

Growth in body length depends on the available food, temperature, and the current body length. If food is not limited, the growth follows a vonBertalanffy growth curve as in Dynamic Energy Budget models (Jager et al., 2013). Apart from that, we model the following fundamental processes feeding, death, birth and immigration. We do not explicitly model emigration. We model population dynamics on a local scale matching the resolution of single empirical observations to better understand under which conditions the large variability of observed salp densities emerges on the observational resolution, while the overall extent of the AMLR survey is in the order of hundred thousand $\mathrm{km}^{2}$ (Reiss et al., 2008).

\subsubsection{Emergence}

The main patterns of intra-annual abundances, inter-annual fluctuations in abundance, body length growth rate distributions, and number of reproductive cycles will emerge from local path dependent indirect interactions between salps through their resource.

\subsubsection{Sensing}

Salps sense the amount of food in the patch they are located in, but only move based on random movements, and only a single patch per day.

\subsubsection{Interaction}

Salps interact indirectly via the food resource.

\subsubsection{Observations}

During the simulation, the abundances of salps are accumulated for each month. For each time step the ratio between blastozooids and oozoids is calculated and recorded (only if oozoids are around to avoid division by zero). Also, the daily body length growth rates of oozoids and blastozooids are stored in a list, but only for the first 5000 days to reduce memory allocation. The peak abundance during a year is also stored. Daily abundances are stored during the summer months (January to March) over all years to compare this distribution with the empirical abundance distribution (see Fig. 6). Finally, the number of full reproductive cycles is stored for each year and the day of the year when migration into the simulation arena occurred.

\subsubsection{Initialization}

The simulation starts during midsummer (January 1), with a daily time step. Since most simulations run for 300,000 consecutive days the choice of the starting date does not affect the results. At the initiation of the simulation the state variable chla is initialized for all patches with a value of $0.5 \mathrm{mg}$ chlorophyll $a \mathrm{~m}^{-3}$. Two oozoids are created with length $2 \mathrm{~cm}$ assuming that these oozoids have not released a chain yet. We initialize the model with oozoids since there is evidence that they are longer lived (up to 24 months, Loeb and Santora, 2012) than blastozooids (up to 7 months, Loeb and Santora, 2012) and that oozoids may overwinter at larger depths (Loeb and Santora, 2012). We do not explicitly model fertilization and assume that single male blastozooids will be present to fertilize the first cohort of released chains.

\subsection{Submodels}

\subsubsection{Growth}

For each salp (each female blastozooid in a chain is modelled as an individual) in the simulated arena the growth is determined for each daily time step. Salps located in the same patch compete for the resource. Potential food uptake (Eq. (1)) of individual salps, ing, is proportional to their surface, $l^{2}$, and food availability, $f$, (with chla: chlorophyll $a$ density in a grid cell in mg chlorophyll $a \mathrm{~m}^{-3}$, and $K$ the half saturation constant) following a Holling type II functionality (Eq. (2)) and a factor, $g$,describing the maximum area specific assimilation rate (see Table 1 for an overview of all parameters and their elasticities):

ing $=g \times f \times l^{2}$

$f=\frac{\text { chla }}{\text { chla }+K}$

Parameters $\left(g=0.0025 \mathrm{mg}\right.$ chlorophyll $a \mathrm{~cm}^{-2}$ and $K=0.2 \mathrm{mg}$ chlorophyll $a \mathrm{~m}^{-3}$ ) have been parameterized that modelled ingestion rates are in good agreement with ingestion rates reported in the literature (von Harbou, 2009, p. 108 and p. 205, Fig. S3). As potentially many individuals compete for the available resource we first calculate the overall food demand in a given patch, $F_{\text {dem }}$. If the food demand

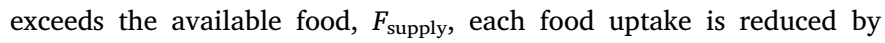
$F_{\text {supply }} / F_{\text {dem }}$ to avoid consuming more resources than are available and to model the interaction as scramble competition in contrast to contest competition (Begon et al., 2006). 
Table 1

Overview of parameters, reference values and their elasticities. Elasticities are computed as $E=\left(\left(y\left(p_{\max }\right)-y\left(p_{\min }\right)\right) / y\left(p_{\text {ref }}\right)\right) / 0.2$, where $p_{\text {min }}$ is the parameter decreased by $10 \%, p_{\max }$ is the parameter increased by $10 \%$ and $p_{\text {ref }}$ is the reference value. The response $y$ has been averaged over 10 simulations. We use the maximum abundance during the simulation to determine $E_{\text {peak }}$ and the median of seasonal peak abundances to determine $E_{m e d i a n}$.

\begin{tabular}{|c|c|c|c|c|c|c|}
\hline Parameter & Parameter & Submodels & Value & Units & Elasticity $E_{p e a k}$ & Elasticity $E_{\text {median }}$ \\
\hline$g$ & Assimilation rate & Growth & 0.0025 & $\mathrm{mg} \mathrm{chl} \mathrm{a/ \textrm {cm } ^ { 2 }}$ & -0.02 & -0.27 \\
\hline$r b_{\text {ref,oozoid }}$ & Oozoid vonBertalanffy growth rate & Growth & 0.024 & $\mathrm{~d}^{-1}$ & 2.20 & 3.80 \\
\hline$r b_{\text {ref,blastozooid }}$ & Blastozooid vonBertalanffy growth rate & Growth & 0.0155 & $\mathrm{~d}^{-1}$ & 2.0 & 3.40 \\
\hline$K$ & Half saturation constant & Growth & 0.2 & $\mathrm{mg}$ chla $\mathrm{m}^{-3}$ & -2.6 & -5.50 \\
\hline$t_{\text {surv }}$ & Starvation & Death & 30 & days & 0.3 & 0.5 \\
\hline$m$ & Daily mortality & Death & 0.025 & - & -3.1 & -3.60 \\
\hline$p_{E}$ & Embryo survival & Reproduction & 0.7 & - & 0.7 & 0.5 \\
\hline$v d$ & Vegetation delay & Primary production & 45 & days & -0.1 & 0.1 \\
\hline$r_{\text {algae, } \max }$ & Rate of primary production & Primary production & 0.25 & $\mathrm{~d}^{-1}$ & 0.9 & 2.9 \\
\hline$d$ & Chl a decay & Primary production & 0.05 & $d^{-1}$ & -0.5 & -2.60 \\
\hline$p_{i}$ & Immigration probability & Immigration & 0.0085 & - & -0.2 & 2.70 \\
\hline$n_{i}$ & Number of immigrants & Immigration & 10 & - & 0.60 & 0.60 \\
\hline$l_{i}$ & Size of immigrants & Immigration & 3 & $\mathrm{~cm}$ & 0.5 & 1.1 \\
\hline
\end{tabular}

The vonBertalanffy growth rate is modelled temperature dependent following the Arrhenius relation:

$r_{B}(T)=r_{B, r e f} \times \exp \left(\frac{T_{A}}{T_{1}}-\frac{T_{A}}{T}\right)$

where $T$, is the temperature in Kelvin, $T_{1}$, is the reference temperature where $r_{\mathrm{B}}\left(T_{1}\right)=r_{\mathrm{B}, \mathrm{ref}}, T_{\mathrm{A}}=8000 \mathrm{~K}$ at $\mathrm{T}_{1}=275 \mathrm{~K}$ is the Arrhenius temperature which is good agreement with reported $\mathrm{Q}_{10}=2.8$ (Iguchi and Ikeda, 2004). Temperature varies between $2{ }^{\circ} \mathrm{C}$ and $-2{ }^{\circ} \mathrm{C}$ from summer to winter (see section 'primary production' for more details). The parameter $r_{B, r e f}$ is different for oozoids and blastozooids $\left(r_{B, \text { ref,oozoids }}=0.024 \mathrm{~d}^{-1}, r_{\mathrm{B}, \text { ref,blastozooids }}=0.0155 \mathrm{~d}^{-1}\right)$. Parameter values are calibrated pattern oriented (see section "parameter calibration" for further details). The growth in body length is modelled following the vonBertalanffy growth curve (Eq. (4)) with functional response, $f$, given in Eq. (2). This functional response may be adjusted if the demand is higher than the supply by the factor $F_{\text {supply }} / F_{\text {demand }}$ as outlined above. The only inputs are the current body length, $l$, and the density of chlorophyll $a$ in the patch. The parameter, $l_{\max }$, gives the asymptotic body length that is assumed to be $l_{\max }=14 \mathrm{~cm}$ for oozoids and $l_{\max }=5 \mathrm{~cm}$ for blastozooids (for individual blastozooids not for chains) for both sexes.

$\frac{\Delta l}{\Delta t}=r_{B}(T) \times\left(f \times l_{\max }-l\right)$

If the increase in length based on Eq. (4) is positive the length of the individual will be updated. If it is negative the length of the individual will not change, but the state variable, $t_{\text {starv, }}$, will be increased by one. Finally, the food in the patch, pchla, will be reduced by each individual by, ing, given in Eq. (1). If the chla density (chla $=$ pchla $/ 16 \mathrm{~m}^{-3}$ ) in a patch drops to zero it will be set to chla $=0.005 \mathrm{mg}$ chlorophyll $a \mathrm{~m}^{-3}$. This reflects some small inflow from outside of organic matter without explicitly modelling a computational expensive diffusion process.

\subsubsection{Reproduction}

The reproductive cycle of salps is relatively complex. Oozoids asexually produce multiple chains of blastozooids that can lead to massive recruitment events. In the model we assume that oozoids start reproduction when they have reached a body length of $l=6 \mathrm{~cm}$ based on empirically estimates of first block releases that ranges from $55 \mathrm{~mm}$ to $70 \mathrm{~mm}$ (Foxton, 1966, von Harbou, 2009). Foxton (1966) concluded from his empirical work that four chains with 750-800 aggregates could be released. This is in accordance with the empirical findings of Daponte et al. (2001) who suggested that under optimal conditions more than 800 aggregates could be released in up to five chains by one oozoid based on specimen ranging from $13 \mathrm{~mm}$ to $95 \mathrm{~mm}$ in size. Given this empirical evidence we have implemented a length dependence reproduction process such that each oozoid releases their first chain with 150 blastozooids when it has grown to $6 \mathrm{~cm}$ or larger. Thus, the second chain with 180 blastozooids will be released if oozoid length is $7 \mathrm{~cm}$, the third chain with 210 blastozooids will be released if oozoid length is $8 \mathrm{~cm}$, and the final fourth chain with 240 blastozooids will be released if the oozoid length is $9 \mathrm{~cm}$. After the release of the fourth chain the oozoid dies in the model assuming that the reproductive inactive oozoid will have a high mortality and to reduce computational operations. Not all oozoids release four chains, since some will die before or never reach the needed body length. In the model the process of chain release will take more than four weeks from the first release to the final chain release. Chains consist of female blastozooids. We assume that all female blastozooids will be fertilized. Thus, each female blastozooid can give birth to one oozoid by sexual reproduction. This is implemented in a way that each female blastozooid is releasing one oozoid once it has reached the body length of $l=2.5 \mathrm{~cm}$ following the observations by Foxton (1966) who found that most embryos are released by blastozooids in the size class of $25-30 \mathrm{~mm}$. We assume that $70 \%$ of all embryos survive based on estimated proportions of failed embryos observed across various latitudes and temperatures (Henschke et al., 2018, Henschke and Pakhomov, 2019). After releasing the embryo, the blastozooid changes sex from female to male and fertilizes other female blastozooids which we do not explicitly model. At this stage we assume that the chains break up and that male blastozooids act as individuals.

\subsubsection{Mortality}

Apart from embryo mortality described in the reproduction paragraph, mortality can occur in three different ways in the model namely by predation, age, and starvation. Predation is implemented as a daily mortality, $m=0.025$. There are no direct measurements of mortality for Salpa thompsoni, but length dependent extrapolations for daily mortality based on experimental work for the smaller salp species Thalia democratica have been suggested (Henschke et al., 2015a, 2015b). Together with considerations of negative density dependence daily mortality rate, $m \sim 0.03 \mathrm{~d}^{-1}$, have been used in a previous salp model (Henschke et al., 2018). Age related mortality is introduced to avoid immortality and is prescribed and set at 500 days, mostly due to a lack of information regarding maximum life span. This prescribed maximum age-related mortality is not a significant source of mortality. Oozoids also die if they have released four chains. Finally, individuals also die if they have not met their food demands for a number of days specified by the parameter, $t_{\text {starv }}=30$ days. Whether food demand is met or not is determined by the vonBertalanffy growth Eq. (4), if the calculated growth increment is negative we assume that the food demand has not been fulfilled. Parameter values have been chosen that the seasonal abundance patterns (observed abundances in summer and 
very low abundances in winter) are well represented and that they are in the ranges of other modelling studies (Henschke et al., 2018). With the given parameterization simulated local abundances go to zero during winter.

\subsubsection{Primary production}

To allow for an interaction between foraging salps and primary production we have included a patch-based conceptual primary production that follows a logistic Eq. (5) where the growth rate $r_{\text {algae }}$ depends on the time of the year (6). As in other population models the conceptual primary production model is based on a logistic equation and the parameters have been calibrated that the observed chlorophyll $a$ content matches the observations (e.g. Ryabov et al., 2017). The change in chla in Eq. (5) depends on the carrying capacity $K=0.675 \mathrm{mg}$ chlorophyll $a \mathrm{~m}^{-3}$, the maximum growth rate, $r_{\text {algae,max }}=0.25$ day $^{-1}$, and the decay rate, $d=0.05$ day $^{-1}$. The carrying capacity has been chosen such as that the peak chlorophyll $a$ content in the model matches the average observed chlorophyll $a$ content chla $=0.54 \mathrm{mg}$ chlorophyll $a \mathrm{~m}^{-3}$. In the scenario where we base our assumption on the chlorophyll availability of the AMLR data we draw the expected chla peak abundance, $N^{*}$, from a lognormal distribution with meanlog $=3.83$ and sdlog $=0.58$ derived from a maximum likelihood estimate of the measured AMLR chlorophyll data presented in Fig. 2b. Carrying capacity is then determined by $K=N \times\left(1-d / r_{\text {algae,max }}\right)$. Doing so the peak chla content will match $N^{*}$ in the absence of salp predation. The seasonal fluctuation of the growth rate $r_{\text {algae }}$ is described in Eq. (6). The maximum growth rate $r_{\text {algae,max }}=0.25$ day $^{-1}$ and, $v d$ (in units of days), parameterizes the time before new year's day when primary production is at its maximum.

$\frac{\Delta \text { chla }}{\Delta t}=r_{\text {algae }}(t) \times$ chla $\times\left(1-\frac{\text { chla }}{K}\right)-d \times$ chla

$r_{\text {algae }}(t)=r_{\text {algae }, \text { max }} \times\left(0.5 \times \cos \left(\frac{t+v d}{365} \times 360\right)+0.5\right)$

Temperature is modelled in a similar conceptual way to reflect intra-annual changes in the simulation arena with a summer temperature of $2{ }^{\circ} \mathrm{C}$ (see Fig. $2 \mathrm{~d}$ for the distribution of observed temperatures between $-1.6{ }^{\circ} \mathrm{C}$ and $4.3{ }^{\circ} \mathrm{C}$ and a mean of $1.6{ }^{\circ} \mathrm{C}$ ) and a winter low of $-2^{\circ} \mathrm{C}$.

$T(t)=\cos \left(\frac{t}{365} \times 360\right) \times 2+273$

Temperature, $\mathrm{T}$, is measured in Kelvin to be consistent with the Arrhenius relation in Eq. (3) and annual temperature varies between its maximum and minimum for four degrees Kelvin.

\subsubsection{Immigration}

During winter the number of salps is getting low and due to demographic stochasticity and starvation local extinction usually occurs in the model. If that happens we model stochastic migration into the local model arena. Therefore, each time step we draw a random number from a uniform distribution [0,1). If the random number is below the immigration probability $\left(p_{i}=0.0085\right)$ we allow $n_{I}=10$ oozoids (body length $l_{I}=3 \mathrm{~cm}$ ) to migrate into the model arena. The migration probability has been chosen after systematic variation of this parameter and comparing with the observed data (see Fig. 6). The chosen immigration probability results in migration events every 118 days on average. The size of the seed population of 10 has been chosen to be able to investigate the question whether local salp blooms can be caused by small initial populations that undergo explosive population growth. The elasticity analyses (Table 1 ) shows that the results do not change substantially if the number of immigrants is slightly varied. The immigrating individuals are randomly distributed in space.

\subsubsection{Movement}

Movement of salps within the model domain is implemented as a random walk to allow salps to distribute themselves in space. Therefore, all salps are randomly moved each time step (day) to one of their eight neighboring patches.

\subsection{Input}

In the variable food scenario, we read in a time series of chlorophyll $a$ values generated from a lognormal distribution that was derived from the observed data (meanlog $=3.83$ and sdlog $=0.58$ ).

\subsection{Parameter calibration and Elasticity}

The model has been calibrated to reproduce the intra- and interannual abundance patterns on the population level. Mortality related parameters have been chosen that the intra-annual pattern of very low abundances during winter and variable abundances in summer could be met. Suitable ranges of the vonBertalanffy growth rates have been determined using the BehaviorSearch tool using genetic algorithms (BehaviorSearch v1.10, Stonedahl and Wilensky, 2010) with an earlier model version to match simulated peak abundances and the observed abundance data. Final calibration of the vonBertalanffy growth rate for oozoids, $\mathrm{rb}_{\text {oozoid, }}$, has been done by choosing a value that matches the median of the observed salp abundances well. The Elasticities of the parameters and their values are given in Table 1. Elasticities report the relative change in a response variable if parameters change. Therefore, we changed all parameters one at a time for $\pm 10 \%$. Elasticities are computed as $E=\left(y\left(p_{\max }\right)-y\left(p_{\min }\right)\right) / y\left(p_{\text {ref }}\right) / 0.2$, with $p_{\min }$ is the parameter decreased by $10 \%, p_{\max }$ is the parameter increased by $10 \%$ and $p_{\text {ref }}$ is the reference value. The response $y$ has been averaged over 10 simulations. We use the maximum abundance during the simulation and the median of seasonal peak abundances as the response variables (see Table 1). The simulation results are most sensitive to changes in the parameters describing the growth rates in body length (oozoid von-

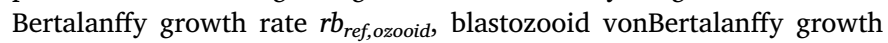

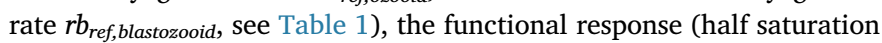
constant $K$, see Table 1 ), and the primary production (Rate of primary production $\left.r_{\text {algae, } \max }\right)$. This highlights the importance of an improved understanding of the physiological processes and primary production especially in a changing environment where empirically measured relationships may change. In addition, changes in daily mortality also change the simulation results substantially (daily mortality $m$, see Table 1). Immigration probability $\left(p_{i}\right)$ does not affect the simulated peak abundance, but the median peak abundance. This finding reflects that the maximum abundance in the model is capped by the food resource.

\section{Results}

The explicit spatial nature of the model shows how strongly the abundance estimates and their variability depend on the resolution of the observations. A snapshot of the model is presented in Fig. 4 for very high salp densities where the mean abundance is 20 salps $\mathrm{m}^{-3}$ on the extent of the simulation arena $\left(2704 \mathrm{~m}^{-3}\right)$. However, the variability of salps abundances on the patch level is between 0 ( $2 \%$ of all patches are empty) and 9920 individuals per patch $\left(=62\right.$ individuals $\left.\mathrm{m}^{-3}\right)$. The fact that female blastozooids are aggregated in chains intensifies this spatial variation. This resolution dependent variability can be shown in a similar way for the chlorophyll $a$ content. The mean concentration is $0.56 \mathrm{mg}$ chlorophyll $a \mathrm{~m}^{-3}$ in the snapshot (Fig. 4b), but ranges from $0.14 \mathrm{mg}$ chlorophyll $a \mathrm{~m}^{-3}$ to $0.86 \mathrm{mg}$ chlorophyll $a \mathrm{~m}^{-3}$ in exploited and unexploited patches, respectively.

The model is able to reproduce empirical patterns such as the ratio between oozoids and blastozooids (both sexes) (Fig. 5a). Since each oozoid can produce several hundred blastozooids during asexual 
a)

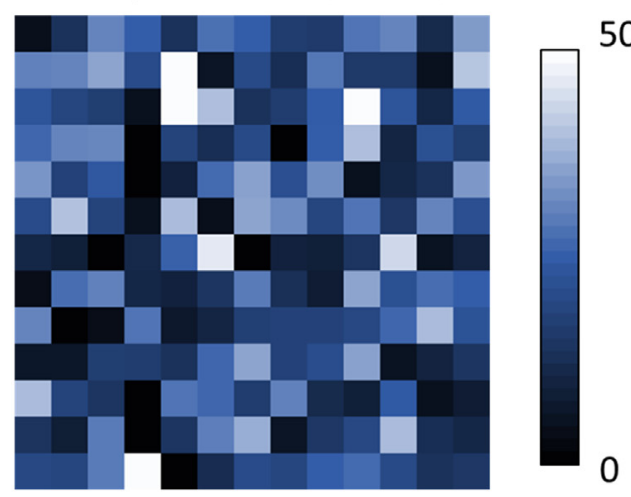

b)

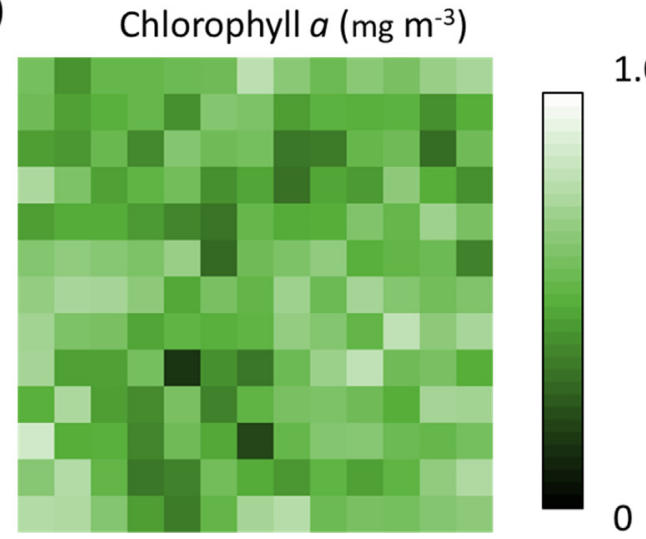

Fig. 4. Typical state of the model during a salp bloom $(\mathrm{N}=54,400)$. Spatial resolution is $16 \mathrm{~m}^{-3}$ and spatial extent is $13 \times 13$ patches. While the average salp density is 20 salps $\mathrm{m}^{-3}$ the density on the resolution of the simulation varies between 0 and 62 salps $\mathrm{m}^{-3}$. The mean chlorophyll $a$ content is $0.56 \mathrm{mg}$ chlorophyll $a \mathrm{~m}^{-3}$. On the grid resolution chlorophyll $a$ content varies between 0.14 and $0.86 \mathrm{mg}$ chlorophyll $a \mathrm{~m}^{-3}$.

reproduction the blastozooid to oozoid ratio increase as high as 5000 in the constant food scenario and to 19,174 in variable food scenario. However, the median value for the blastozooid to oozoid ratio is 43 and 4 for the constant food scenario and the variable food scenario matching observed ratios between 4 and 100 (Pakhomov and Hunt, 2017). In the constant food environment daily growth in length of blastozooids is also very variable ranging from $0 \mathrm{~mm} \mathrm{~d}^{-1}$ to $0.5 \mathrm{~mm}$ $\mathrm{d}^{-1}$ with a mean of $0.2 \mathrm{~mm} \mathrm{~d}^{-1}$. Daily growth of oozoids is substantially larger varying from $0 \mathrm{~mm} \mathrm{~d}^{-1}$ to $2.3 \mathrm{~mm} \mathrm{~d}^{-1}$ with an average of $1 \mathrm{~mm} \mathrm{~d}^{-1}$. In the food variable scenario the growths rates are more variable for oozoids $\left(0-2.8 \mathrm{~mm} \mathrm{~d}^{-1}\right.$, average: $\left.1.2 \mathrm{~mm} \mathrm{~d}^{-1}\right)$ and blastozooids $\left(0-0.6 \mathrm{~mm} \mathrm{~d}^{-1}\right.$, average: $\left.0.2 \mathrm{~mm} \mathrm{~d}^{-1}\right)$. The simulated intraannual abundance distribution is consistent with overall expectation that salps are mostly present in the Southern Ocean during austral summer, as found in observations (Foxton, 1966, Atkinson et al., 2017). In the model, the number of reproductive cycles (Fig. 5d) ranges from 0 (reproductive failure) to 2.5. Simulated salp abundances during the summer months vary over four orders of magnitude, similar to the observations in the AMLR data. The distribution of salp abundances depends on the immigration probability (Fig. 6a). In the constant food scenario the observed maximum abundances cannot be reproduced (Fig. 6a). For the variable food scenario the maximum observed abundances can be simulated and the results are less sensitive to changes in the immigration probability matching the observed distribution well (Fig. 6b). Simulated peak abundances vary substantially between years (see Fig. S2) and the resulting peak abundance in a season depends very much on the time when the seeding population migrates into the local model arena (Fig. 7a). Only if the seed

population migrates into the domain during early spring the very high abundances can be achieved the following summer (Fig. 7a). If we define a salp bloom as abundances larger or equal to the $95 \%$ quantile of observed salp abundances $\left(q=1.4\right.$ salps $\left.\mathrm{m}^{-3}\right)$ we can simulate the bloom risk for different immigration probabilities (Fig. 7b). Bloom risk as defined above (more than 1.4 salps $\mathrm{m}^{-3}$ ) increases with immigration probability, however, the risk rises more slowly in the variable food scenario and converges at a lower level. In the constant food scenario, the bloom risk grows up to 0.8 with immigration probability $=1$ (not shown in the graph).

\section{Discussion}

We have developed an individual-based model for salps that is able to resolve physiological and demographic processes to better understand the population dynamics of salps and its potential for their explosive population growth. The model reproduces empirical patterns on several organizational levels. Starting from the individual level modelling daily growth rates, our model results (variable food scenario: growth rates for oozoids: mean $=1.2 \mathrm{~mm} \mathrm{~d}^{-1}$, $\mathrm{sd}=1 \mathrm{~mm} \mathrm{~d}^{-1}$ and $\max =2.8 \mathrm{~mm} \mathrm{~d}^{-1}$ ) are consistent with the latest empirical observations (Pakhomov and Hunt, 2017) reporting $2.83 \pm 0.42 \mathrm{~mm} \mathrm{~d}^{-1}$ for oozoid daily growth and cohort-modelling results $\left(0.7-2 \mathrm{~mm} \mathrm{~d}^{-1}\right.$, Henschke et al., 2018). It is worth noting that growth rates in the simulation were measured all year round and often included zero growth due to possible severe competition or low productivity during winter. On the observational scale the grazing impact of salps is often described as moderate (e.g. Pakhomov et al., 2002) although it has been stated that salps can consume more than $100 \%$ of the primary production (Dubischar and Bathmann, 1997). On small spatial and temporal resolution salps may be food limited negatively affecting their growth.

Older estimates of daily growth rates based on cohort analyses by Loeb and Santora (2012) were substantially lower for oozoids (growth rates for oozoids: mean $0.23 \pm 0.04 \mathrm{~mm} \mathrm{~d}^{-1}$ ) and higher than the simulated average growth rate $\left(0.2 \mathrm{~mm} \mathrm{~d}^{-1}\right)$ for blastozooids $\left(0.4 \pm 0.05 \mathrm{~mm} \mathrm{~d}^{-1}\right)$. The lower estimate for oozoid growth rate has profound consequences for the estimates of one full reproduction cycle duration suggested to be 9-12 months (Foxton, 1966, Loeb and Santora, 2012). However, if we use parameterizations resulting in daily growth rates comparable with those reported by Loeb and Santora (2012) the salp peak abundances could never exceed 0.5 individuals $\mathrm{m}^{-3}$ even if the most positive assumptions for migration (immigration probability $100 \%$ ) and food availability (variable food scenario) were used (see Fig. S1). This is in line with a previous modelling study where a scenario based on the growth rate estimates by Loeb and Santora (2012) resulted in non viable salp populations (Henschke et al., 2018). Assuming low growth rates salp blooms in our model would require a massive immigration or potential accumulation of oozoids over at least two years, which was in fact suggested by Loeb and Santora (2012). Indeed, if a substantial fraction of the salp population would survive beyond summer/fall, it could act as the starting ground for a salp bloom in the next year. This could not be possible in our model because in our simulation salp abundances during winter get very low. Thus, salp blooms only occurred if a successful migration of a seed population started in early spring. Whether the early year migration could potentially be interpreted as salps surviving from the previous season at greater depths or suitable adjacent habitats still requires confirmation. Interestingly the simulated median of the peak abundance for the slow growth $\left(0.03\right.$ individuals $\left.\mathrm{m}^{-3}\right)$ is in the same order of magnitude as the observed median of 0.06 individuals $\mathrm{m}^{-3}$ from the AMLR sites. This highlights the importance of probabilistic risk assessments instead of comparing statistics such as the median. Another mechanism that could lead to massive salp blooms is the aggregation of salps from a large spatial domain by physical forces. To clarify the importance of this process model of ocean currents and turbulences and individual-based models need to be coupled (Dorman et al., 2011). 
a)

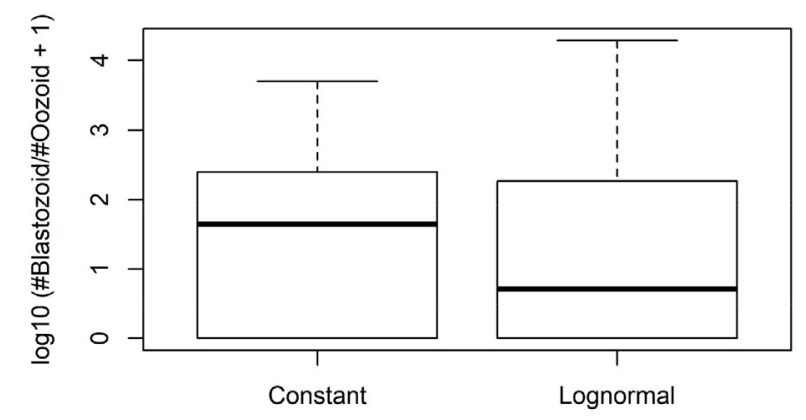

c)

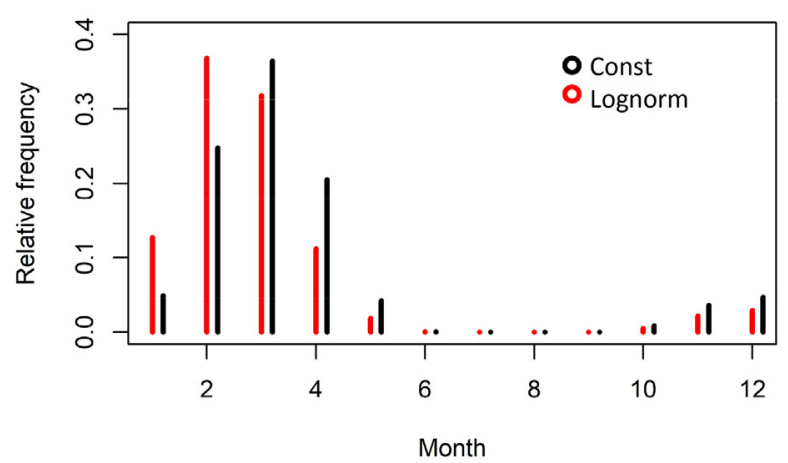

b)

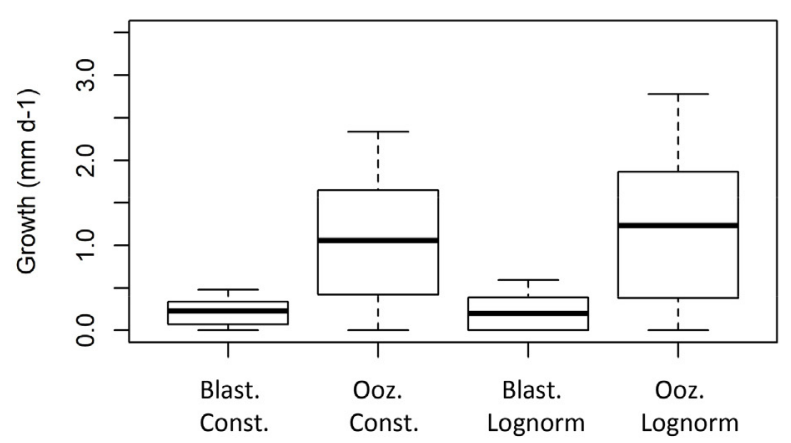

d)

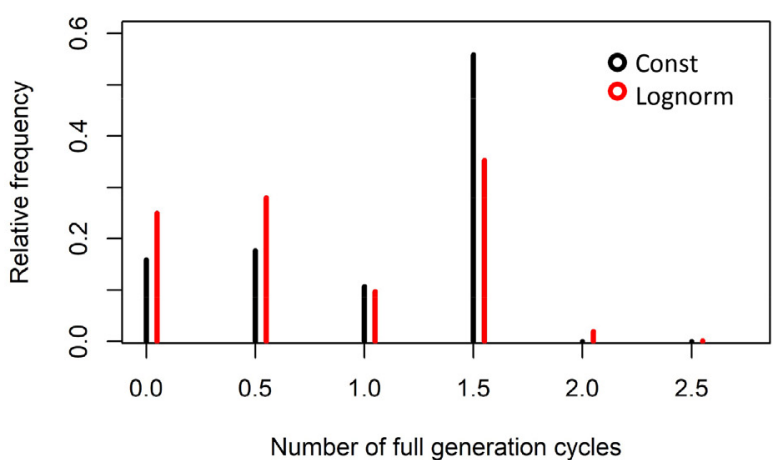

Fig. 5. Diagnostic patterns produced by the model. (A) On average more blastozooids than oozoids are present in the system (the median ratios between blastozooids and oozoids are 43 for the constant chlorophyll $a$ scenario and 4 for the variable food (lognormal) scenario). (b) Daily growth rates covering the range reported in the literature. (c) Intra-annual distributions are in line with the fact that salps are only expected in the Antarctic Summer around the Western Antarctic Peninsula. (d) The distribution of fully completed life cycles ranges from 0 to 2.5 .

Our simulations highlight the variability in the population of salps that are caused by two sources of uncertainty: environmental stochasticity (variability in the primary production) and demographic stochasticity caused by random death events and immigration of small seeding populations. Our results show that if maximum primary production does not change from year to year, the abundance of salps is mainly driven by the immigration (or population survival during overwintering) of small seeding populations. With global change intrusion of warm waters transporting salps southward may become more frequent potentially resulting in more salp blooms (Loeb et al., 1997, Pakhomov et al., 2002). To better understand changes in southward transportation of salps coupling of advection models and salp models is needed on larger spatial scales similar to the modelling of krill larvae transport and distribution (Mori et al., 2019). The increase in the risk of salp blooms, however, is modified by other environmental factors, which can be seen in our simulations when maximum primary production is drawn from a lognormal distribution. In that case, salp blooms may be locally prevented in years of lower primary production. This highlights the importance of forcing models with values drawn from the empirical distributions on the relevant scale for demographic and physiological processes, rather than average values over large spatial and temporal scales. Improved environmental models are needed that can predict how temporal and spatial variability of chlorophyll $a$ will vary with global climate change to project the population dynamics and the probability of salp blooms. To upscale local results to biogeographical regions is challenging since crucial information such as primary productivity and sea ice coverage will be only available on spatial resolutions that are not necessarily matching the spatial resolution of the relevant demographic and physiological processes (Levin, 1992, Chave, 2013). In particular, the non-linear relationship between food availability and duration of sea ice cover on growth and reproduction will require suitable transfer functions to link the observation and process scales (Bloschl and Sivapalan, 1995). Therefore, the linkage of improved ecosystem models with higher spatial resolution and spatial explicit ecological models will be highly beneficial especially if the biotic interaction between krill and salps are to be considered (Elith and Leathwick, 2009, Schurr et al., 2012).

\section{Conclusions}

With an individual-based local salp model we have explored how the huge variability in observed salp abundances can be better understood. We have found that fluctuations in environmental conditions together with demographic processes such as the migration of small seed populations can cause variability in the observed range on the local scale. For large salp blooms successful immigration in early spring is required. This may require that oozoids have successfully overwintered in the area (Loeb and Santora, 2012) and that sea ice retreat started early which is assumed to favor salp blooms (Atkinson et al., 2004). However, the overwintering hypothesis is difficult to support since no salp monitoring campaigns have been conducted during winter in the AMLR area yet (Atkinson et al., 2017). It is suggested that the migration of salps together with the intrusion of warm waters or/and salp winter non-extinction events will become more probable (Loeb et al., 1997, Pakhomov et al., 2002). The increase in the risk of salp blooms due to migration (or/and winter survival) will still be buffered to some extent by environmental stochasticity of primary productivity. These findings highlight the importance of considering the large variability in the population dynamics of salps together with heterogeneous environmental conditions in space and time to understand the risk of salp blooms in the Southern Ocean. 
a)

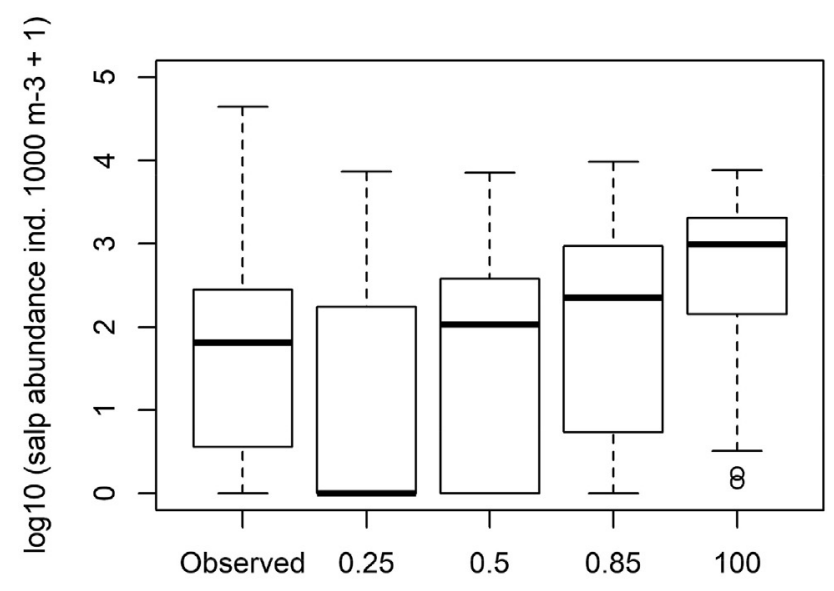

Immigration probability (\%)

b)

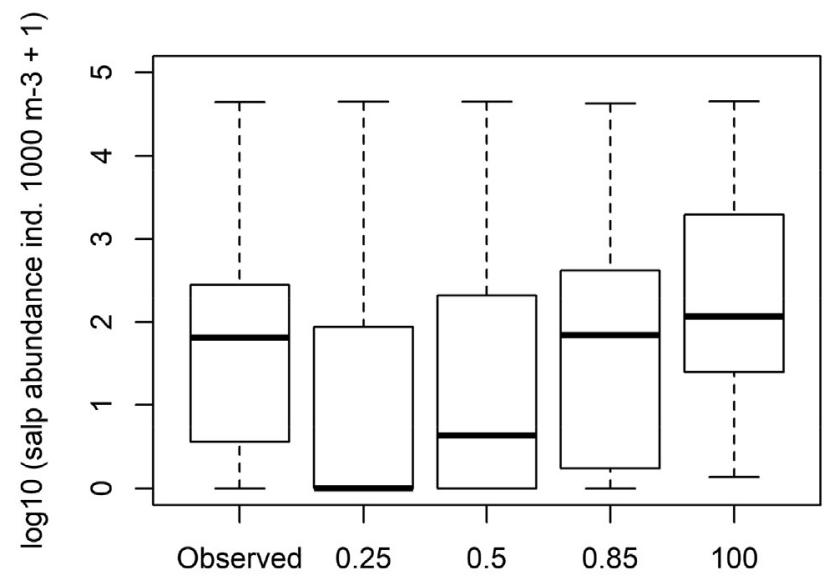

Immigration probability (\%)

Fig. 6. Comparing distributions of observed salp abundances and simulated daily summer month abundances (January-March) for 800 simulated years. The simulated abundances strongly depend on the immigration probability. (a) In the scenario with constant food availability the maximum observed salp density cannot be reached. (b) Only in the variable chlorophyll a scenario (lognormal) observed maximum abundances can be achieved.

\section{Declaration of Competing Interest}

The authors declare that they have no known competing financial interests or personal relationships that could have appeared to influence the work reported in this paper.

\section{Acknowledgements}

We thank two anonymous reviewers for their insightful and constructive comments. This work was supported by the Federal Ministry of Education \& Research BMBF Germany Project PEKRIS (The PErformance of Krill and Salps to withstand in a warming Southern Ocean) awarded to JG (03F0746B) and BM (03F0746A). We also thank the German Academic Exchange Service DAAD (Project 57386792) for funding travelling for BM and JG to partially support this work. We gratefully acknowledge the support of the German Centre for Integrative Biodiversity Research (iDiv) Halle-Jena-Leipzig, funded by the German Research Foundation (FZT 118). a)

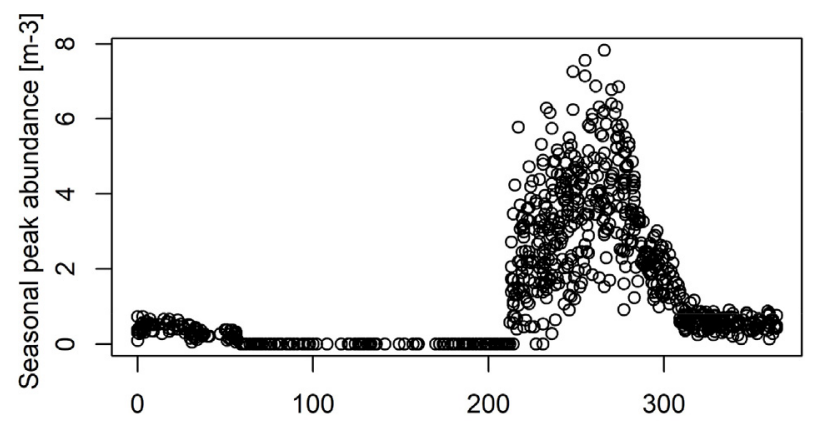

Immigration day in the season

b)

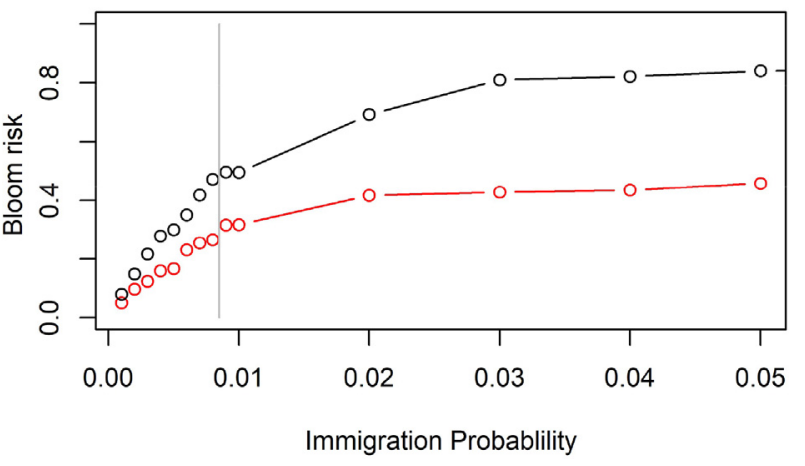

Fig. 7. (a) Maximum peak abundances in the season strongly depend on the timing when a seeding population has successfully migrated into the local area. Only if migration happens in early spring massive blooms can be expected in the following summer (simulations have been made with the constant food scenario). (b) The risk for a salp bloom (here defined as the $95 \%$ quantile of observed salp densities which is 1.4 salps $\mathrm{m}^{-3}$ ) is higher for the constant chlorophyll $a$ scenario (black line) and can partly be buffered by heterogeneities in the chlorophyll $a$ content (red line). The gray line indicates the immigration probability that resulted in the best match with the observed salp abundance distributions. (For interpretation of the references to colour in this figure legend, the reader is referred to the web version of this article.)

\section{References}

Atkinson, A., et al., 2017. KRILLBASE: a circumpolar database of Antarctic krill and salp numerical densities, 1926-2016. Earth Syst. Sci. Data 9 (1), 193-210.

Atkinson, A., Siegel, V., Pakhomov, E., Rothery, P., 2004. Long-term decline in krill stock and increase in salps within the Southern Ocean. Nature 432 (7013), 100-103.

Begon, M., Townsend, C.R., Harper, J.L., 2006. Ecology: From Individuals to Ecosystems. Blackwell Publishing Ltd, USA.

Bloschl, G., Sivapalan, M., 1995. Scale issues in hydrological modeling - a review. Hydrol. Process. 9 (3-4), 251-290.

Chave, J., 2013. The problem of pattern and scale in ecology: what have we learned in 20years? Ecol. Lett. 16, 4-16.

Daponte, M.C., Capitanio, F.L., Esnal, G.B., 2001. A mechanism for swarming in the tunicate Salpa thompsoni (Foxton, 1961). Antarct. Sci. 13 (3), 240-245.

Dorman, J.G., Powell, T.M., Sydeman, W.J., Bograd, S.J., 2011. Advection and starvation cause krill (Euphausia pacifica) decreases in 2005 Northern California coastal populations: Implications from a model study. Geophys. Res. Lett. 38. https://doi.org/10. 1029/2010GL046245.

Dubischar, C.D., Bathmann, U.V., 1997. Grazing impact of copepods and salps on phytoplankton in the Atlantic sector of the Southern Ocean. Deep-Sea Res. Part II-Top. Stud. Oceanogr. 44 (1-2), 415-433.

Elith, J., Leathwick, J.R., 2009. Species Distribution Models: Ecological Explanation and Prediction Across Space and Time. Annu. Rev. Ecol. Evol. Syst. 40, 677-697.

Foxton, P., 1966. The distribution and life history of Salpa thompsoni Foxton with observations on a related species, Salpa gerlachei Foxton. Discov. Rep. 34, 1-116.

Grimm, V., et al., 2006. A standard protocol for describing individual-based and agentbased models. Ecol. Model. 198 (1-2), 115-126.

Grimm, V., Berger, U., DeAngelis, D.L., Polhill, J.G., Giske, J., Railsback, S.F., 2010. The ODD protocol A review and first update. Ecol. Model. 221 (23), 2760-2768.

Henschke, N., Smith, J.A., Everett, J.D., Suthers, I.M., 2015a. Population drivers of Thalia democratica swarm: insights from population modelling. J. Plankton Res. 37, 1074-1087.

Henschke, N., Everett, J.D., Richardson, A.J., Suthers, I.M., 2016. Rethinking the Role of 
Salps in the Ocean. Trends Ecol. Evol. 31 (9), 720-733.

Henschke, N., Pakhomov, E.A., Groeneveld, J., Meyer, B., 2018. Modelling the life cycle of Salpa thompsoni. Ecol. Model. 387, 17-26.

Henschke, N., Smith, J.A., Everett, J.D., Suthers, I.M., 2015b. Population drivers of a Thalia democratica swarm: insights from population modelling. J. Plankton Res. 37 (5), 1074-1087.

Henschke, N., Pakhomov, E.A., 2019. Latitudinal variations in Salpa thompsoni reproductive fitness. Limnol. Oceanogr. 64, 575-584.

Huntley, M.E., Sykes, P.F., Marin, V., 1989. Biometry and trophodynamics of Salpathompsoni foxton (tunicata, thaliacea) near the antarctic peninsula in austral summer, 1983-1984. Polar Biol. 10 (1), 59-70.

Iguchi, N., Ikeda, T., 2004. Metabolism and elemental composition of aggregate and solitary forms of Salpa thompsoni (Tunicata: Thaliacea) in waters off the Antarctic Peninsula during austral summer 1999. J. Plankton Res. 26, 1025-1037.

Jager, T., Martin, B., Zimmer, E.I., 2013. DEBkiss or the quest for the simplest generic model of animal life history. J. Theor. Biol. 328, 9-18.

Levin, S.A., 1992. The problem of pattern and scale in ecology. Ecology 73 (6), 1943-1967.

Loeb, V., Siegel, V., Holm-Hansen, O., Hewitt, R., Fraser, W., Trivelpiece, W., Trivelpiece, S., 1997. Effects of sea-ice extent and krill or salp dominance on the Antarctic food web. Nature 387 (6636), 897-900.

Loeb, V., Hofmann, E.E., Klinck, J.M., Holm-Hansen, O., 2010. Hydrographic control of the marine ecosystem in the South Shetland-Elephant Island and Bransfield Strait region, $57,519-542$.

Loeb, V.J., Santora, J.A., 2012. Population dynamics of Salpa thompsoni near the Antarctic Peninsula: Growth rates and interannual variations in reproductive activity (1993-2009). Prog. Oceanogr. 96 (1), 93-107.

Mori, M., Corney, S.P., Melbourne-Thomas, J., Klocker, A., Kawaguchi, S., Constable, A., Summer, M., 2019. Modelling dispersal of juvenile krill released from the Antarctic ice edge: Ecosystem implications of ocean movement. J. Mar. Syst. 189, 50-61.

Nicol, S., Foster, J., 2016. The Fishery for Antarctic Krill: Its Current Status and Management Regime. In: Siegel, V. (Ed.), Biology and Ecology of Antarctic Krill.
Springer International Publishing, pp. 387-421.

Pakhomov, E.A., Froneman, W., Perissinotto, R., 2002. Salp/krill interactions in the Southern Ocean: spatial segregation and implications for the carbon flux. Deep-Sea Res. Part II-Top. Stud. Oceanogr. 49 (9-10), 1881-1907.

Pakhomov, E.A., Hunt, B.P.V., 2017. Trans-Atlantic variability in ecology of the pelagic tunicate Salpa thompsoni near the Antarctic Polar Front. Deep-Sea Res. Part II-Top. Stud. Oceanogr. 138, 126-140.

R Core Team, 2019. R: A language and environment for statistical computing. R Foundation for Statistical Computing, Vienna, Austria. URL https://nam03.safelinks. protection.outlook.com/?url $=$ https $\% 3 \mathrm{~A} \% 2 \mathrm{~F} \% 2 \mathrm{Fwww} . \mathrm{r}$-project.org $\% 2 \mathrm{~F} \&$ amp;data $=02 \% 7 \mathrm{C} 01 \% 7 \mathrm{CMurugaR} \% 40$ ReedElsevier.com \%7C40232d542c894b4fbf8c08d7fb09a206\%

7C9274ee3f94254109a27f9fb15c10675d\%7C0\%7C0\%7C637253891563620494\& amp;sdata = YKmUL1\%2BRQK\%2FhqT\%2FBASUmT9UKG6kRH0qxsUqFdWNsxr0\% 3D\&amp;reserved $=0$.

Reiss, C.S., Cossio, A.M., Loeb, V., Demer, D.A., 2008. Variations in the biomass of Antarctic krill (Euphausia superba) around the South Shetland Islands, 1996-2006. ICES J. Mar. Sci. 65 (4), 497-508.

Ryabov, A.B., de Roos, A.M., Meyer, B., Kawaguchi, S., Blasius, B., 2017. Competitioninduced starvation drives large-scale population cycles in Antarctic krill. Nat. Ecol. Evol. 1 (7). https://doi.org/10.1038/s41559-017-0177.

Schurr, F.M., et al., 2012. How to understand species' niches and range dynamics: a demographic research agenda for biogeography. J. Biogeogr. 39 (12), 2146-2162.

Stonedahl, F., Wilensky, U., 2010. BehaviorSearch [computer software]. Center for Connected Learning and Computer Based Modeling. Nortwestern University,

Evanston, IL. http://www.behaviorsearch.org.

von Harbou, L., 2009. Trophodynamics of Salps in the Atlantic Southern Ocean University of Bremen. University of Bremen Ph.D.

Wilensky, U., 1999. NetLogo. http://ccl.northwestern.edu/netlogo/. Center for Connected Learning and Computer-Based Modeling. Northwestern University, Evanston, IL. 